\title{
"Persistence" in rats: Effects of testosterone
}

\author{
WILLIAM R. THOMPSON and JANET S. WRIGHT \\ Queen's University at Kingston, Kingston, Ontario, Canada
}

\begin{abstract}
We present two experiments designed to examine further the reported effect of the steriod hormone testosterone on persistence. Persistence was here defined in terms of difficulty of shifting from one discrimination habit to a new one in which previously relevant dimensions now were irrelevant. In the first study, adult male rats injected with a moderate dosage level of testosterone showed increased persistence compared with oil-injected and androsteroneinjected groups and with animals receiving a high dosage of testosterone. In a second study, male rats injected with the antiandrogen cyproterone acetate showed a reduction in persistence compared with controls. In both experiments, arousal levels were measured by nonreinforced barpress rates during and between trials. In neither study were differences in arousal level relevant to the drug effects. The results are in general agreement with those of other workers using chicks and mice.
\end{abstract}

During the last few years, a slender but important, body of research has been concerned with the effects of the steroid hormone testosterone on behaviors considered to reflect the hypothetical construct "persistence"' (see Andrew, 1976; Archer, 1976; Rogers, 1976). The term "persistence" has been used to summarize the characteristics of "search" patterns observed in young and adult chickens given injections of testosterone. These characteristics are described by Andrew (1972b, p. 193) as follows: "increased persistence of activation or availability of search specifications, once they [have] come into use." In the typical situations used, treated animals are found to be more distractible than controls in searching for food by alterations in stimuli "relevant" to the search, though less distractible by "nonrelevant" stimulus changes (Archer, 1974). The opposite effect has been produced by the androgen inhibitor cyproterone acetate (Rogers, 1974). It is not entirely clear what the defining criteria of "relevance" are, though operationally the latter appears to refer to the degree of spatial proximity to operandum stimuli, for example, a food dish, a runway, or a food-grain color in an arena test (Archer, 1974, 1977). Thus, elevated testosterone levels might be thought of as producing a narrowing or focusing of attention.

It is noteworthy that the testosterone effect has not been found in female animals (chicks), even though the latter show other changes such as in body weight and comb growth (Andrew, 1972a). Further, even in males, the attentional changes occur well before the

\footnotetext{
The above research was supported by a grant from the Queen's Advisory Research Committee, Life Sciences Subcommittee. The authors thank Dr. N. L. Freedman of the Psychology Department and Professor H. Jellinck of the Biochemistry Department for useful advice. Requests for reprints should be sent to the first author.
}

morphological effects. This has led Andrew to suggest that the action of testosterone on persistence is "physiological" and may involve central androgensensitive receptor sites. He has further speculated that, from a biological point of view, the kinds of changes produced by increasing testosterone should be adaptive in male animals at the onset of breeding, since they might be expected to enhance territorial and mate acquisition and retention, and defensive capabilities.

Andrew (1972a) and Archer (1977) have rejected the possibility that merely changes in arousal level are involved by reason of the following facts: (1) The persistence effect is found over a wide range of fooddeprivation schedules (Andrew \& Rogers, 1972); (2) extinction rates of treated males are the same as those of untreated males and females (Rogers, 1974); and (3) strains of mice known to differ in arousal level or emotionality do not differ in respect to induced persistance of search (Archer, 1977).

The possibility of linkage between a relatively simple change in hormone level and such a complex behavior as persistence is of great interest and importance. However, in the view of the writers, the data base for this relation needs to be made more precise. At least in some of the studies referred to above, parameters are not clearly defined, effects of different variables are confounded, and behavioral measures are often qualitative and of indeterminate reliability. In addition, it needs to be extended. Subjects have mostly been young chicks, two exceptions being in the study of Rogers (1974) on adult chickens and in that of Archer (1977) on adult (60-day) mice.

The major purpose of the present studies was to examine the effects of testosterone using a more precise measure of persistence. This was essentially an extradimensional discrimination shift in which a set of cues relevant in an initial learning task became 
irrelevant in a second learning task and those irrelevant before became relevant. Persistence was operationally defined as retardation of capacity to make the shift normally accomplished by ignoring the previously relevant cues. Details of the procedure are outlined below. They seem to the authors to embody the essential features of the various situations used by Andrew and his colleagues, though, in our view, allowing much greater precision and control. However, we also fully recognize that, given the ambiguity of the concept of persistence, our specification of it may still be somewhat arbitrary.

In addition, we attempted also (1) to extend the generality of the effects to that most popular of laboratory animals, the adult rat; (2) to employ a more exact and direct control for possible steroid-produced changes in behavioral arousal; (3) to narrow inferences about the specificty of testosterone action by use of a related androgen compound, androsterone, and (4) to examine the effects on our measure of the androgen inhibitor, cyproterone acetate.

\section{EXPERIMENT 1}

\section{Method}

Subjects. Forty male Charles River Long-Evans hooded rats (Canadian Breeding Laboratories, Toronto) were used. They were approximately 60 days old at the start of the experiment and their average weight was $225 \mathrm{~g}$. They were housed in individual cages and maintained on a partial deprivation schedule of three 5-g pellets of Purina Chow per day and unlimited access to water for 5 days prior to training.

Apparatus. Training was conducted in a double-barred Skinner box $40 \times 38 \times 50 \mathrm{~cm}$ constructed of Plexiglas on the top and ends, aluminum on the two sides, and a grid floor. Bars were located on one wall, $5 \mathrm{~cm}$ apart, with an automatically recessible food cup between them. Fourteen centimeters above the food cup was a yellow trial-indicator light (General Electric 1829), which signaled availability of reinforcement. A white cue light (General Electric 1829) was located $8 \mathrm{~cm}$ over each bar. The apparatus was housed in a small sound-shielded room. During formal testing, all stimulus parameters were under automatic control by a digital logic BRS rack (Electrocraft of Canada) located in a separate room. Reinforcement consisted of $.3 \mathrm{ml}$ of sweetened condensed milk diluted with two parts of water.

Procedure. The animals were initially gentled and habituated to the apparatus. Following this phase, they were next shaped by hand to barpress and to discriminate on from off periods as signaled by the center light. When an animal pressed the bar which had previously been designated as correct (randomly, left or right), the food cup was manually presented for $7 \mathrm{sec}$. Following this, the cup was retracted for $8 \mathrm{sec}$, and then a new trial begun. Trials continued until the subjects had responded correctly in 5 of the 15 daily trials and withheld a barpress response during the 8 -sec intertrial interval for 5 consecutive trials. This stage of training was accomplished for all rats over an average period of 8 days.

In the next phase, formal discrimination learning was begun and controlled automatically by the BRS rack. Two programs were used. In the light discrimination program (LDP), the correct bar was indicated by illumination of the cue light over it, with position (left or right) randomized from trial to trial. In the position discrimination program (PDP), bar position was the relevant dimension, with either left or right bar (randomly assigned) being correct. Cue lights were lighted on each trial, but randomly in respect to position. In both programs, the sequence of events for all single trials was as follows: (1) Onset of trial-indicator light (indicating reinforcement available) coincident with the onset of a cue light; both lights were terminated by first correct barpress up to a maximum trial length of $60 \mathrm{sec}$. (2) Food cup with reinforcement presented at correct barpress up to a maximum of $7 \mathrm{sec}$, then recessed. (3) Eight-second intertrial interval. (4) Repeat of above cycle. Maximum duration for a daily test was $45 \mathrm{~min}$. Criterion for learning was set at 10 consecutive correct responses. Subjects had been randomly assigned to one of the two program sequences prior to control or steroid treatments. Following the latter, they received the alternate program. Both correct responses and nonreinforced presses during and between trials were recorded. After reaching the learning criterion on the initial program, the animals were assigned randomly to one of four groups as described next.

Treatment groups. Group T25 rats were injected with a $25-\mathrm{mg} /$ 100-g-body-weight dosage of testosterone enanthate (Sigma Chemical Co., St. Louis) dissolved in .1 ml of sesame oil. Group T55 subjects received $55 \mathrm{mg} / 100 \mathrm{~g}$ body weight of androsterone (Sigma Chemical Co., St. Louis) in .1 ml sesame oil alone. All injections were administered subcutaneously in the nape of the neck. Dosage levels were chosen to be roughly equivalent to those used by Andrew and his colleagues, as well as on the basis of initial pilot work. Since testosterone is released slowly from an oil vehicle (Junkman, 1952), testing was not resumed until $72 \mathrm{~h}$ postinjection. Criterion of posttreatment learning was again set at 10 consecutive correct responses.

\section{Results}

The measure of persistence was given essentially by the difference between pre- and posttreatment trials to criterion. The measures of arousal were total number of nonreinforced barpresses during and between trials. These measures of persistence and arousal will be considered separately.

Persistence. The result of major interest relates to the interaction between treatment groups and pre- vs. postinjection performance. The data are presented in Figures 1A and 1B. Regardless of order of programs, the T25 group took more than double the number of trials to reach criterion in postinjection testing $[F(3,32)=140.91, p<.001]$. Other groups, as shown by Newman-Keuls tests, did not differ from each other. However, all were significantly different from the T25 group in postinjection performance. This massive effect on both shifts plus differential learning rates by the other groups on the two sequences were mainly responsible for the main-effect differences between drug groups $[\mathrm{F}(3,32)=37.28$, $\mathrm{p}<.001]$, pre- vs. postinjection performance $[\mathrm{F}(1,32)$ $=157.06, \mathrm{p}<.001]$, and the interaction between programs and pre- vs. postinjection trials $[\mathrm{F}(1,32)=$ $38.37, \mathrm{p}<.001$ ]. In addition, program PDP (position) was learned more easily than program LPD (lights) $[\mathrm{F}(1,32)=13.79, \mathrm{p}<.001]$. The main result, however, is remarkably clear. The T25 animals showed a marked impairment in their ability to perform the extradimensional shift regardless of order of programs.

Arousal. Analysis of nonreinforced barpresses 

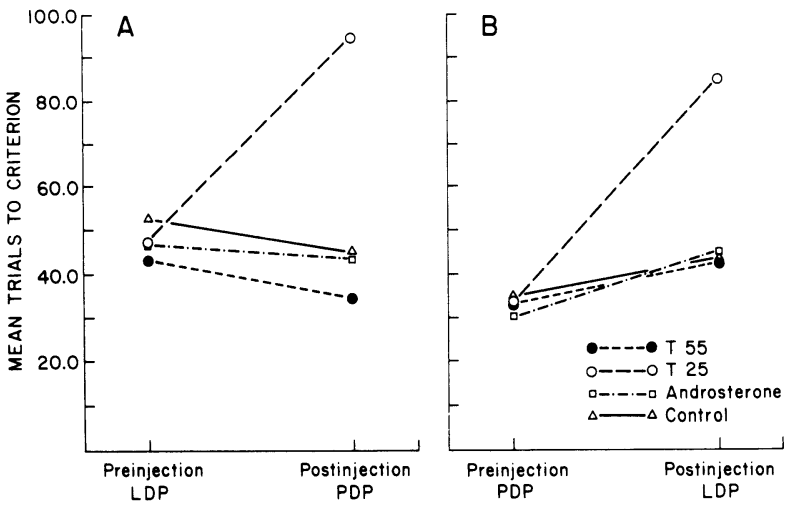

Figure 1. (A) Pre- and postinjection trials to criterion in the testosterone (T25, T55), androsterone (A), and control (C) groups for program sequence LDP-PDP. (B) Pre- and postinjection trials to criterion in the testosterone (T25, T55), androsterone (A), and control (C) groups for program sequence PDP-LDP.

during trials yielded only one significant result, namely the interaction between program and pre- vs. postinjection performance $[\mathrm{F}(1,32)=15.38, \mathrm{p}<$ $.001]$. More presses were made in program LPD than in program PDP preinjection. Likewise, for barpresses between trials, all subjects scored higher postinjection than preinjection $[\mathrm{F}(1,32)=6.03, \mathrm{p}<.05]$. Since there were no differences for either measure between drug groups either as a main effect or in combination with other variables, it is clear that the results on the persistence measure were probably not a function of activation level.

\section{EXPERIMENT 2}

\section{Method}

Subjects. Twenty male Chester Beatty rats (Biological Breeders, Ottawa) were used. They were approximately 60 days of age and had an average weight of $225 \mathrm{~g}$ at the start of testing. Maintenance was the same as in Experiment 1.

Apparatus. The same equipment and reinforcement procedures were used as in the previous experiment.

Procedure. Initial training procedures were the same as outlined in Experiment 1. Following preinjection, test subjects were randomly assigned to one of two groups.

Treatment groups. Group Cyp A subjects received subcutaneous injections of $1.0 \mathrm{mg} / 100 \mathrm{~g}$ body weight of cyproterone acetate (Schering, A.G., Berlin) dissolved in $.02 \mathrm{ml}$ of propylene glycol: castor oil (1:5 ratio) daily for 9 days. Control rats were injected with the vehicle only in the same amount over the same period of time. On the 10th day postinjection, the animals were tested on the new program. Measures of persistence and arousal were the same as those used in Experiment 1.

\section{Results}

Persistence. Analysis of trials to criterion yielded a significant interaction between treatment groups and pre- vs. postinjection $[\mathrm{F}(1,16)=39.89, \mathrm{p}<.001]$, with the experimental group showing significantly better performance than controls in the postinjection task. These data collapsed across programs are shown in Figure 2. This large interaction is probably responsible for a main effect difference between the two groups $[\mathrm{F}(1,16)=5.59, \mathrm{p}<.05]$. Program PDP was learned more readily than program $\operatorname{LPD}[\mathrm{F}(1,16)$ $=6.77, \mathrm{p}<.05]$, particularly in the preinjection phase $[F(1,16)=98.88, p<.001]$. Likewise, most animals performed better in the postinjection test $[F(1,16)=$ 45.14, $\mathrm{p}<.001$ ], though this improvement showed up mainly in the Cyp A group animals $[F(1,16)=39.89$, $\mathrm{p}<.001]$.

Arousal. No significant effects were found in respect to barpresses during trials. For barpresses between trials, analysis revealed higher scores postinjection $[\mathrm{F}(1,16)=6.69, \mathrm{p}<.05]$ and a significant triple interaction that is not readily interpretable.

Thus the effects of cyproterone acetate on persistence also appear to be largely independent of activation level as measured.

\section{DISCUSSION}

It is very clear from the data that testosterone at the 25-mg dosage level depressed capacity for making an extradimensional discrimination shift which is our operational definition of an increase in persistence. This effect was reasonably specific, since the control steroid-androsterone-produced no such deficit. Further, the effect was not produced by changes in arousal level which was constant (as measured here) across all groups. In addition, the antiandrogen, cyproterone acetate, was found to decrease persistence. Thus, the main results corroborate those of Andrew and other workers cited in the introduction. However, there still remain some puzzles.

In the first place, it is not known just what factors were responsible for the change in persistence of the T25 group. Qualitatively, however, we observed the

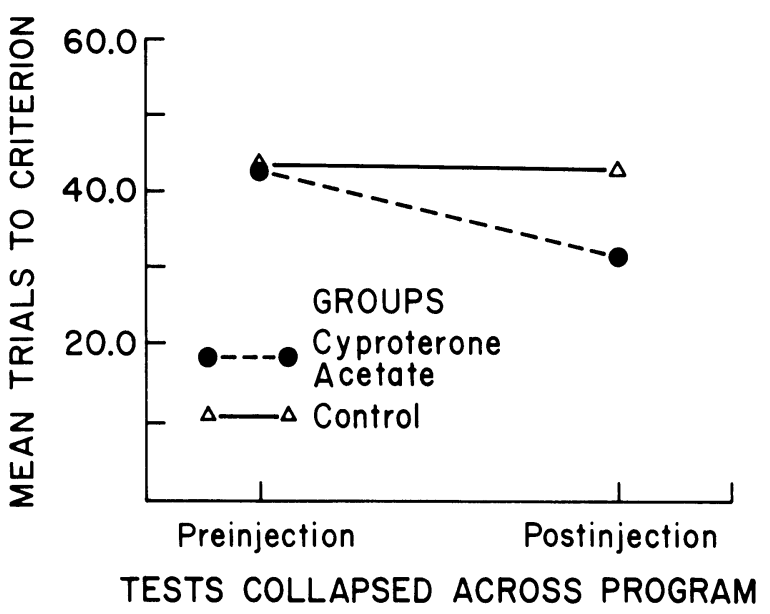

Figure 2. Pre- and postinjection trials to criterion on the cyproterone acetate and control groups collapsed across programs. 
following: Rats of the other three groups, when faced with the new program, would continue responding to the previously relevant cues until, at some point, lack of reinforcement produced a complete cessation of responding followed by exploration of the test chamber. Eventually, rats would then resume responding and discover the correct new strategy. Subjects in the T25 group showed the same basic pattern, but extended it in time-particularly the exploration phase during which they tended to press randomly regardless of whether the trial light was on or not. However, it is uncertain whether their systematic persistence in the old habit was greater than controls or not. What is clearly called for is a fine-grain on-line record of behavior during the shift phase. This is now being undertaken by one of the authors in a new set of studies. Hopefully, such data will aid in sharpening up our definition of persistence. Although the term no doubt has intuitive appeal, it is at present too coarse to allow exact hypothesis testing.

The second problem relates to the behavior of the T55 group which performed the shift as rapidly as the control groups. This is a surprising result. However, it is of some interest to note that in one of Andrew's (1972a) studies, high-dosage testosterone $(25 \mathrm{mg})$ subjects characteristically showed less persistence by several measures than medium-dosage $(12.5 \mathrm{mg})$ animals. Likewise, they showed less marked body-weight increases and comb growth and lower copulation and attack-pecking than medium-dosage chicks. These differences were apparently not statistically significant. However, they are suggestive of a turning downward of the dose-response curve, as found in our study. Our result might have several explanations. Testosterone at both 25 and $55 \mathrm{mg} / 100 \mathrm{gm}$ body weight are pharmacological rather than physiological dosage levels. It may be that the 55-mg dosage represents a concentration too high to be out of solution within the $72 \mathrm{~h}$ between time of injection and time of postinjection testing. Alternately, high dosages may be taken up more slowly by receptor sites in the hippocampus or preoptic areas (Pasqualani, 1976). Another hypothesis is that at higher dosage levels some deactivation processes commence to occur more strongly or more rapidly, thereby canceling out any behavioral effect. These possibilities could be examined by varying the injection-test interval. In any event, it seems clear that precise dose-response curves need to be established. This is also being undertaken by one of the authors. When this is accomplished, it may then be feasible to turn to the more physiological problems of sites and rates of uptake of testosterone.

A final problem should be mentioned. In view of the results obtained with the androsterone group, we inferred that the effects on persistence produced by testosterone were specific to that compound. However, it must be emphasized that androsterone is a much weaker agent than testosterone. Thus, it is possible that at a higher dosage level than that used here, it might well produce an effect.

In summary, our results basically agree with those reported by Andrew and his colleagues. However, they are derived from procedures having more methodological precision and extend to another mammalian taxon. Our data have also generated a number of hypotheses, some of which we are presently engaged in testing.

\section{REFERENCES}

Andrew, R. J. Changes in search behavior in male and female chicks, following different doses of testosterone. Animal Behaviour, 1972, 20, 741-750. (a)

ANDREw, R. J. Recognition processes and behavior with special reference to effects of testosterone on persistence. In D. S. Lehrman, R. A. Hinde, \& E. Shaw (Eds.), Advances in the study of behavior. New York \& London: Academic Press, 1972. (b)

Andrew, R. J. Attentional processes and animal behavior. In R. A. Hinde \& P. P. G. Bateson (Eds.), Growing points in ethology, Cambridge: Cambridge University Press, 1976.

Andrew, R. J., \& Rogers, L. Testosterone, search behavior and persistence. Nature (London), 1972, 237, 343-346.

ARCHE R, J. The effects of testosterone on the distractibility of chicks by irrelevant and relevant novel stimuli. Animal Behaviour, 1974, 22, 397-404.

Archer, J. Biological explanations of psychological sex differences. In B. B. Lloyd \& J. Archer (Eds.), Exploring sex differences. London \& New York: Academic Press, 1976.

Archer, J. Testosterone and persistence in mice. Animal Behaviour, 1977, 25, 479-488.

Junkman, K. Long-acting androgens. Archives of Experimental Pathology and Pharmacology, 1952, 215, 85-92.

PASQUAlani, J. Receptors and mechanisms of action of steroid hormones (Part 1). New York: Dekken, 1976.

ROGERS, L. J. Persistence and search influenced by natural levels of androgens in young and adult chickens. Physiology \& Behavior, 1974, 12, 197-204.

Rogers, L. J. Male hormones and behavior. In B. B. Lloyd \& J. Archer (Eds.), Exploring sex differences. London \& New York: Academic Press, 1976.

(Received for publication February 22, 1979; revision accepted June 26, 1979.) 